\title{
Design, simulation and experimental verification of heat transfer structure in thermoelectric cooling helmets
}

\author{
Zhaoshen $\operatorname{Tian}^{1,2}, \mathrm{Ju} \mathrm{Xu}^{1,2, *}$ \\ ${ }^{1}$ Institute of Electrical Engineering Chinese Academy of Sciences, Beijing, China \\ ${ }^{2}$ University of Chinese Academy of Sciences, Beijing, China
}

\begin{abstract}
ABSRTACT. In order to solve the problem of sunstroke for helmet wearing person under strong sunshine or close hot environments due to the high temperature between the person's head and the helmet, a thermoelectric cooling helmet is proposed. However, due to the curve nature of the head, the even temperature distribution inside the helmet is normally a big challenge, we propose a thermoelectric cooling helmet using thermoelectric cooler (TEC) combining a new heat transfer design scheme. In this design, using hydrogel with very high percentage of water filled in soft package attached on high thermal conductive thin fins is applied to establish a heat transfer channel between the head and the cold surface of the thermoelectric cooler. The control of the cold surface temperature of the thermoelectric cooler element and the rapid heat transfer of the high thermal conductive thin fins provide precise control of the internal temperature of the helmet, while enhancing the comfort of the helmet by utilizing the softness of the hydrogel. The heat transfer structure of this combination of high thermal conductive thin fins and hydrogel is a very important guide for the heat dissipation of other curved heat generating objects.
\end{abstract}

\section{Introduction}

The relevant literature shows that the head's heat production is large and head is a high heat dissipation zone of the human body ${ }^{[1][2]}$. In the summer heat, takeaway, errands and other staff in the process of outdoor work, in order to ensure safety, will wear a helmet, the current safety helmet generally use the following design scheme: helmet mainly consists of shell (helmet outermost hard shell), cap body (helmet internal foam inner layer), safety harness, brim, air holes, padding composition ${ }^{[3]}$. This helmet design has poor heat dissipation. To solve this problem, it is necessary to achieve good heat dissipation and temperature control inside the helmet. The common physical refrigeration techniques are: phase change refrigeration, gas expansion refrigeration, and thermoelectric refrigeration ${ }^{[4]}$. Thermoelectric cooler is simple in structure, small and lightweight, works without moving parts, produces no vibrations or noise, and can control temperature precisely through a control circuit, reacts quickly to reach the set temperature in a short period of time. It can be seen that thermoelectric cooler is suitable for temperature control inside helmets compared with other methods.

The heat source inside the helmet is the head, and the head is a typical curved heat-generating object, in practice, the curved heat-generating object mostly uses air cooling means to dissipate heat. When the head wears a helmet, the inner space of the helmet is relatively confined, and convective heat dissipation cannot be realized. Therefore, a thermoelectric cooler to control the temperature inside the helmet is the best option to establish a cooling channel between the cold surface of the thermoelectric cooler and the head, and a water cycle cooling structure design is proposed for a thermoelectric cooling helmet using water for cooling ${ }^{[5]}$.

Based on the structure, this paper proposes a heat transfer structure that combines high thermal conductive thin fins and hydrogel. The high thermal conductive thin fins are made of metal with high thermal conductivity and play the role of heat transfer and heat distribution. Hydrogel is a kind of material with water as the dispersion medium, and has a mesh cross-linked structure, the structure of hydrogel is stable, has good physical flexibility, high water content of hydrogel has the characteristics of high heat capacity. The hydrogel has good flexibility and can fit curved objects well to reduce air thermal resistance. Through simulation experiments, the structure of high thermal conductive thin fins and hydrogels is optimized, and the physical model is established and verified according to the optimized structure.

\section{The thermoelectric cooling helmet' design principle}

\subsection{Structure design}

The thermoelectric cooling helmet is a kind of heat transfer structure which applies the thermoelectric cooler to the inside of the helmet, and establishes the high

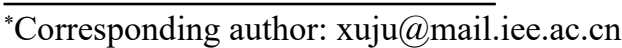


thermal conductive thin fins and the hydrogel between the helmet liner (the surface which is in direct contact with the head) and the cold surface of the thermoelectric cooler, by controlling the temperature at the cold surface of the thermoelectric cooler, the temperature inside the helmet can be controlled.

This paper proposes to build a heat transfer structure inside a thermoelectric cooling helmet with a combination of high thermal conductive thin fins and hydrogel. As shown in the figure 1, the head heat is first transferred to the hydrogel, through which it is transferred to the high thermal conductive thin fins, which ultimately transfer the heat to the cold surface of the thermoelectric cooler. A patent has now been filed for a thermoelectric cooling helmet that uses a water circulation structure for head cooling and temperature control ${ }^{[6]}$. A brief analysis of the two heat transfer structures is presented in Table 1 as follows.

Table 1. A brief analysis of the two heat transfer structures.

\begin{tabular}{|l|l|l|}
\hline & Water circulation structure & $\begin{array}{l}\text { High thermal conductive thin fins and hydrogel } \\
\text { structure }\end{array}$ \\
\hline $\begin{array}{l}\text { Structural } \\
\text { analysis }\end{array}$ & $\begin{array}{l}\text { The heat transfer structure consists of a micro-pump, } \\
\text { water pipe, pump control module, and } \\
\text { cold- water reservoir. The heat transfer structure is } \\
\text { complex. }\end{array}$ & $\begin{array}{l}\text { The heat transfer structure consists of high thermal } \\
\text { conductive thin fins, hydrogels. The heat transfer } \\
\text { structure is simple. }\end{array}$ \\
\hline $\begin{array}{l}\text { Heat } \\
\text { transfer } \\
\text { analysis }\end{array}$ & $\begin{array}{l}\text { The heat transfer is achieved through the circulation } \\
\text { of water to achieve an even heat distribution, heat } \\
\text { resistance between the water pipe and the head is } \\
\text { large. }\end{array}$ & $\begin{array}{l}\text { High thermal conductive thin fins are used for heat } \\
\text { transfer and heat distribution; hydrogel with high } \\
\text { water content has the function of cold storage and } \\
\text { is filled between the heat distribution fins and the } \\
\text { head to reduce thermal resistance. }\end{array}$ \\
\hline $\begin{array}{l}\text { Energy } \\
\text { consumptio } \\
\text { n analysis }\end{array}$ & $\begin{array}{l}\text { Power supply module: micro pump, thermoelectric } \\
\text { cooler, intelligent control module. The water pump } \\
\text { causes an overall increase in the overall energy } \\
\text { consumption of the helmet. }\end{array}$ & $\begin{array}{l}\text { Power supply module: thermoelectric cooler, } \\
\text { intelligent control module. Low energy } \\
\text { consumption, high endurance. }\end{array}$ \\
\hline
\end{tabular}

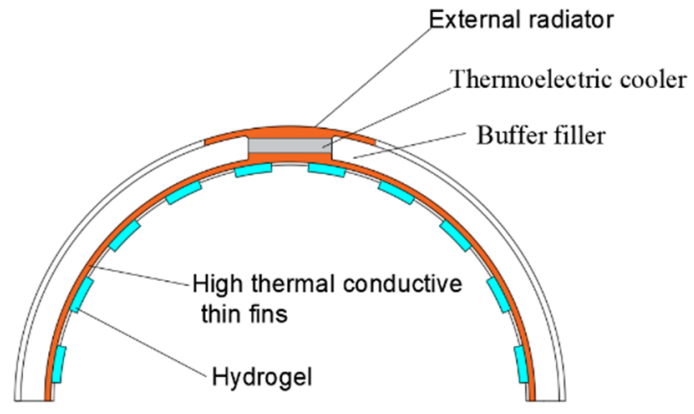

Figure 1. Helmet with high thermal conductive thin fins and hydrogel structure.

According to the table 1 , it can be seen that the proposed heat transfer structure of the combination of high thermal conductive thin fins and hydrogel has the advantages of simple structure, good heat transfer effect, no energy drive, etc. This structure has great reference value for the promotion of thermoelectric cooling helmet application.

\subsection{Analysis of thermal resistance}

Based on the design structure, draw a topological network model of the thermal resistance between the thermoelectric cooler and the head, as shown in the figure 2.

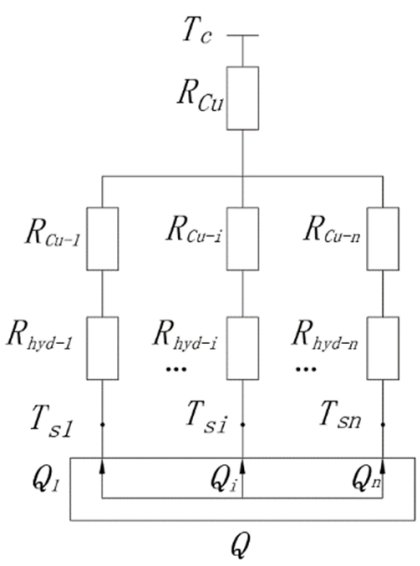

Figure 2. The topological network model of the thermal resistance.

According to figure 2, the expression for the temperature $T_{s i}$ at each point of the head is written as follows:

$$
\begin{gathered}
T_{s i}=T_{c}+Q_{i} *\left(R_{C u-i}+R_{h y d-i}\right)+Q * R_{C u} \\
Q=\sum_{1}^{n} Q_{i}
\end{gathered}
$$

In the formula, $T_{c}$ is the temperature of the cold surface of TEC, $R_{C u-i}, R_{h y d-i}$ is the thermal resistance of the copper heat conduction branch and the hydrogel, $R_{C u}$ is the thermal resistance of the copper heat conduction trunk, $Q$ is the total heat of the head, ignoring the thermal resistances between the connecting layers. $R_{C u-i}, R_{h y d-i}$ and $R_{C u}$ represent the performance parameters of heat transfer structure. Because of the complexity of heat transfer structure and the huge amount of calculation between formulas, the feasibility of this scheme is verified by simulation experiment. 


\section{3 calculation of heat dissipation $Q$ of the head and heat dissipation area $S$}

To study the heat transfer structure through simulation calculations, the heat dissipation must first be determined. Based on the contents of this paper, the heat dissipation $Q$ ${ }^{[7]}$ of the head and the heat dissipation area $S{ }^{[8]}$ are investigated. The heat dissipation of the head $Q$ is about $12.75 \mathrm{~W}$, the heat dissipation area $S$ is about $0.06 \mathrm{~m}^{2}$, and skin thickness of the head is $5 \mathrm{~mm}$. The heat dissipation of the head needs to set a certain margin, so the heat dissipation of the head is set at $20 \mathrm{~W}$.

\section{3-D topological structure model and simulation optimization}

\subsection{Heat transfer structure design}
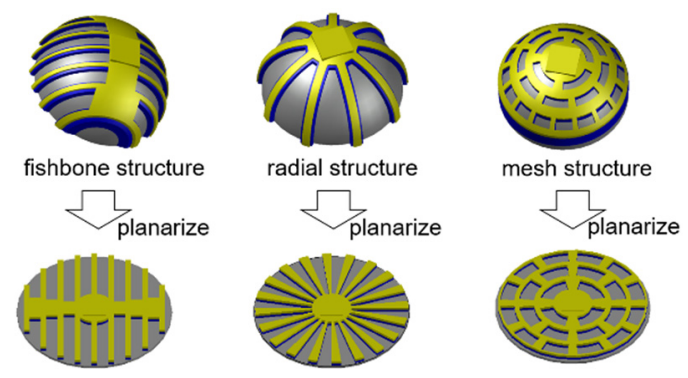

Figure 3. Three heat transfer structures.

A heat transfer scheme using high thermal conductive thin fins combined with hydrogels is used to create a cooling channel between the head and the cold surface of the thermoelectric cooler. In this paper, three structures are designed, a fishbone structure, a radial structure, and a mesh structure. In order to facilitate the modeling, this paper planarizes the three designs with the same amount of high thermal conductive thin fins and hydrogels, and simulate the planarized structure. The planarized heat transfer structure is shown in the figure as an example of a radial structure as shown in figure 4 . The best heat transfer structure is selected by building a model for simulation experiment, and the best heat transfer structure is built for physical verification.

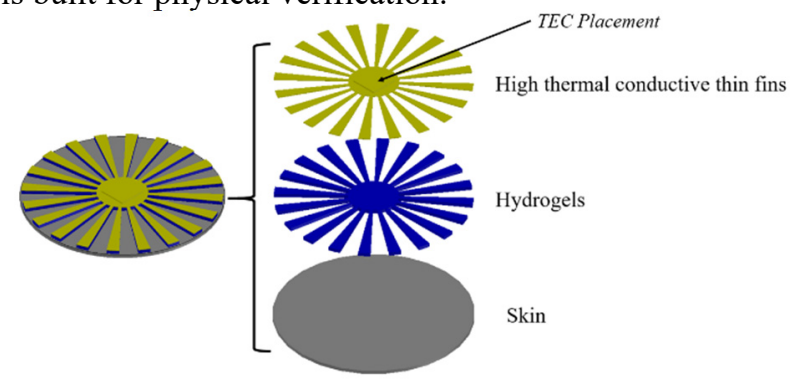

Figure 4. Radial transfer structures.

\subsection{Setting of boundary conditions and thermodynamic parameters}

Heat transfer consists mainly of heat conduction, thermal convection and thermal radiation. The interior space of the helmet is relatively airtight and there is no thermal convection. The effect of thermal radiation is small and negligible.

The simulation model is built based on the material thermal properties of the heat transferring fins and hydrogels in the heat transfer structure given in the table 2 . The boundary conditions are set so that convective heat transfer and radiant heat dissipation are not considered, and the initial temperature of the simulation experiment is $30^{\circ} \mathrm{C}$. The high thermal conductive thin fins are made of copper with a thickness of $1 \mathrm{~mm}$ and the hydrogel is $5 \mathrm{~mm}$.

Table 2. The material thermal properties.

\begin{tabular}{|c|l|l|}
\hline Material & Copper & Hydrogel \\
\hline Density / $\left(\mathrm{kg} / \mathrm{m}^{3}\right)$ & 8960 & 1000 \\
\hline $\begin{array}{c}\text { Specific heat capacity / [ J/ } \\
(\mathrm{kg} * \mathrm{~K})]\end{array}$ & 285 & 4180 \\
\hline $\begin{array}{c}\text { Thermal Conductivity /[W/ } \\
(\mathrm{K} * \mathrm{~m})]\end{array}$ & 400 & 0.6 \\
\hline
\end{tabular}

\subsection{Results and analysis}

With the heat dissipation $Q$ of the head and a constant temperature of $5 \mathrm{~mm}$ inside the skin, temperature simulations of the three design configurations were performed using COMSOL by setting the temperature variation at the cold surface of TEC. The simulation results of the radiation structure are shown in figure 5 . The simulation results of three design schemes are compared. According to the simulation results, the average skin surface temperature, the average temperature of the contact surface between skin and hydrogel, and the temperature difference between skin and hydrogel are plotted and analyzed respectively.

Analysis of the simulation results: simulation and analysis of the three design structures, the average skin surface temperature, the average temperature of the contact surface between skin and hydrogel, and the temperature difference between the contact surface between skin and hydrogel, together verify that the radial structure design has the best heat transfer and heat distribution effect.

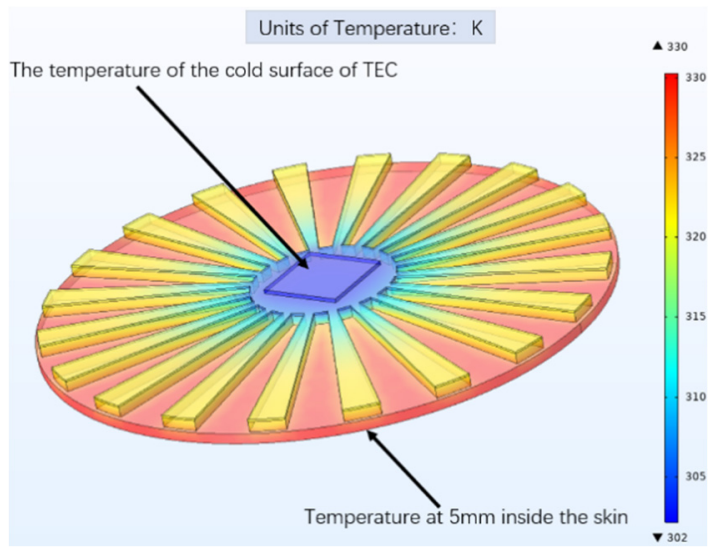

Figure 5. Temperature Simulation Results for Radiation Structures 


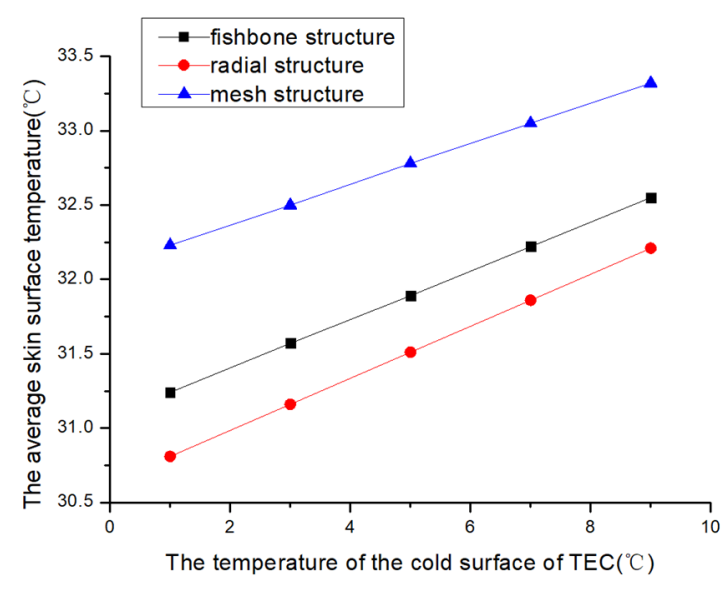

Figure 6. The average skin surface temperature.

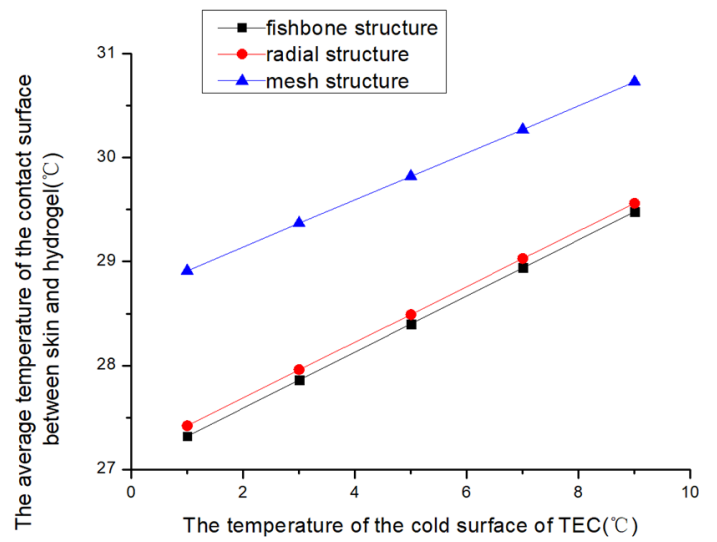

Figure 7. The average temperature of the contact surface between skin and hydrogel.

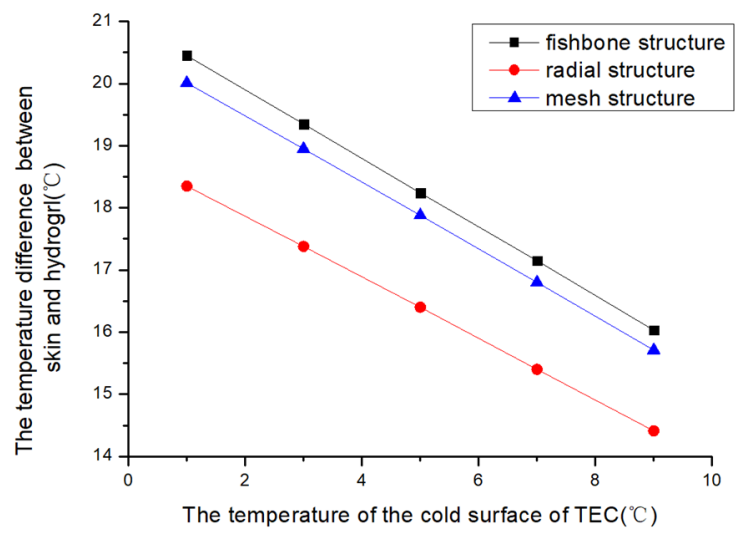

Figure8. The temperature difference between skin and hydrogel.

\subsection{Physical Verification Experiments}

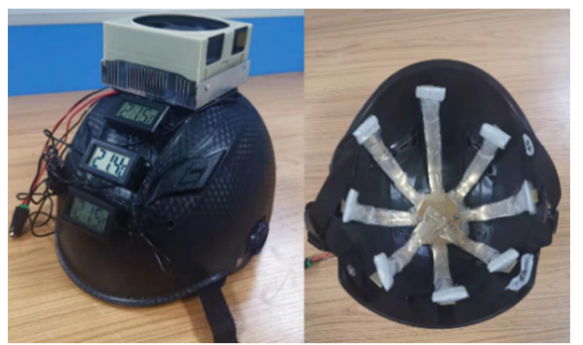

Figure 9. Mock-up of helmet.
To establish a physical model of the radial structure, a heat transfer structure combining high thermal conductive thin fins and hydrogel is added inside the helmet, as shown in the figure 9, wearing a normal helmet for 30 minutes, the acquisition temperature is $31^{\circ} \mathrm{C}$, turn on the thermoelectric cooler, after 15 minutes the acquisition temperature drops to $29^{\circ} \mathrm{C}$. The experiment proves the feasibility of the heat transfer structure with the combination of high thermal conductive thin fins and hydrogel.

\section{Conclusion}

In conclusion, this paper aims at the structural design of the heat transfer structure of the combination of high thermal conductive thin fins and hydrogel between the head and the cold surface of the TEC, and carries out thermodynamic modeling and simulation of the three designed structures. According to the results of the simulation, select the radial structure as the best heat transfer structure, and establishes the physical model according to the radial structure, and verifies the heat transfer structure of the combination of high thermal conductive thin fins and hydrogel by means of crossvalidation between simulation results and experiments. The ability to meet the heat transfer requirements between the head in the helmet and the cold surface of the thermoelectric cooler demonstrates the feasibility of the combined fin and hydrogel heat transfer structure. The combination of high thermal conductive thin fins and hydrogel allows the application of thermoelectric coolers not to be limited to flat surfaces, but rather to create a good heat transfer structure between curved and irregular objects and the thermoelectric cooler, increasing the range of thermoelectric cooler applications.

\section{References}

1. Cheng Pang. Dingliang Gu, Head Cooling in Hyperthermia. J, Space medicine \& medical Engineering, 8(1),67 70(1995).

2. Chunyv Yv. Jiale Jie, et al, Study on the Body Surface Temperature changing with the Indoor Environmental Temperature, J, Spectroscopy and Spectral Analysis, 37(1), 172-176 (2017).

3. GB 811-2010, Helmets for motorcyclists[S].

4. Wei Zhao. Pin Zhang, Research and Application of Thermoelectric Refrigeration Technology, J, REFRIGERATION,39(03),18-21(2020).

5. R.J. Buist, G.D. Streitwieser, The thermoelectrically cooled helmet, Proceeding of the Seventeenth International Thermoelectric Conference, Arlington, (1988).

6. Donglang Wang, Refrigerated Helmet [P]. CN104758115A,2015-07-08.

7. Dahai Xu. Rong Zhu, The sensation of human to temperature \& humility \& wind speed and the cooling, J, Journal OF APPLIED METEOROLOGICAL SCIENCE, 11(04), 430-439. (2000).

8. Chunyu Yu. Jiale Xie. Bin Fei. Junju Zhang, The 
Effect of Ambient Temperature on Human Head's

Surface Skin Temperature, J, Spectroscopy and Spectral Analysis, 37(01),172-176. (2017) 